\title{
Evaluation of Novel Obesity- and Lipid-Related Indices as Predictors of Abnormal Glucose Tolerance in Chinese Women with Polycystic Ovary Syndrome
}

Qianqian Yin ( $\nabla$ w85225569@163.com )

Xuzhou Central Hospital https://orcid.org/0000-0002-9185-7004

Xiaonan Yan

Xuzhou Central Hospital

Yijuan Cao

Xuzhou Central Hospital

Jianhua Zheng

Xuzhou Central Hospital

\section{Research Article}

Keywords: abnormal glucose tolerance, Chinese visceral adiposity index, visceral adiposity index, lipid accumulation product, triglyceride glucose index, polycystic ovary syndrome

Posted Date: February 9th, 2022

DOI: https://doi.org/10.21203/rs.3.rs-1301637/v1

License: (c) (i) This work is licensed under a Creative Commons Attribution 4.0 International License.

Read Full License 


\section{Abstract \\ Purpose}

We aimed to evaluate the performance of Chinese visceral adiposity index (CVAI), visceral adiposity index (VAl), lipid accumulation product (LAP), triglyceride glucose (TyG) as indices in screening abnormal glucose tolerance (AGT) in Chinese women with polycystic ovary syndrome (PCOS), using the oral glucose tolerance test (OGTT) as a reference test. In addition, we essentially compared the abilities of these indices with body mass index (BMI), waist circumference (WC), fasting plasma glucose (FPG).

\section{Materials and Methods}

All 1113 PCOS patients evaluated in this study underwent OGTTs. The 2-h post-oral glucose load (2 h-PG) level was used to categorize subjects into two groups: those having AGT or normal glucose tolerance (NGT) levels.

\section{Results}

A statistically significant positive correlation between levels of $2 \mathrm{~h}-\mathrm{PG}$ and FPG, BMI, WC, LAP, VAl, CVAl, TyG, $(P<0.05)$, was observed. The strongest correlation was found between the levels of $2 \mathrm{~h}-\mathrm{PG}$ and CVAl $(r=0.469)$. The CVAI provided the highest area under the receiver-operating characteristic curve (AUC) for AGT, followed by LAP, BMI, TyG, VAI, WC, and FPG. The CVAl of 32.61 (with AUC: 0.758 , sensitivity: $73.4 \%$, specificity: $69.9 \%$ ) was found to be the cut-off point for AGT in Chinese women with PCOS.

\section{Conclusions}

The CVAI may be used as a screening indicator of AGT in Chinese women with PCOS when there is no time or possibility to perform an OGTT.

\section{Introduction}

Type 2 diabetes mellitus (T2DM), an established risk of cardiovascular disease, disability, and premature death, has become a major worldwide public health burden in the past decade [1,2]. Impaired glucose tolerance (IGT), at an early stage in the natural history of T2DM, may remain underdiagnosed since it is usually asymptomatic, and its detection necessitates an oral glucose tolerance test (OGTT) [3]. Adoption of suitable lifestyle modifications or pharmacological interventions may delay or prevent deterioration of IGT to T2DM [3]. Therefore, a need for earlier detection of IGT has been significantly highlighted.

Polycystic ovary syndrome (PCOS), a very common endocrine disorder, impacts $5-10 \%$ of women in their reproductive age [4]. A more meaningful screening for abnormal glucose tolerance (AGT) is warranted in 
women with PCOS as they show an increased prevalence of disturbances of glucose metabolism compared with the general population and have shown characteristically postprandial abnormalities in glucose metabolism[5-7].

However, OGTT, considered the gold-standard in detecting AGT, is an inconvenient and time-consuming procedure. Therefore, it should have a simple and effective screening tool for AGT in women with PCOS.

Studies have firmly established that both obesity and dyslipidemia are the traditional risk factors for T2DM because these two conditions can increase peripheral tissue insulin resistance (IR) [8]. Therefore, in general populations of different races, several obesity- and lipid-related indices, including visceral adiposity index (VAl) [9-11], lipid accumulation product (LAP) [9,12,13], and triglyceride Glucose index (TyG) $[9,11,14]$, have been commonly suggested as promising surrogate indicators of both T2DM and prediabetes. Recently, the Chinese visceral adiposity index (CVAl), a tool developed to estimate visceral obesity of Chinese individuals, has been suggested as a better predictor of T2DM and prediabetes than any other obesity index for Chinese adults $[1,2,15,16]$.

To our knowledge, women with PCOS have excessive visceral fat accumulation than those without PCOS, even if they are of normal weight, and visceral adiposity is more associated with obesity-related metabolic abnormalities than subcutaneous or peripheral fat accumulation [17]. Therefore, the aforementioned indicators may have different implications for screening of AGT among the PCOS population than in the general population.. Moreover, although there are many studies on the relationships of these indices with T2DM and prediabetes, however, the data establishing an association between these indices and AGT remains scarce $[8,12,18]$, especially for the PCOS population $[19,20]$. Thus, we aimed to evaluate the performance of these indices as tools in screening AGT in Chinese women with PCOS, using the OGTT as a reference.

\section{Material And Methods}

\section{Subjects and study design}

A total of 1113 women with PCOS (aged 13-41 years) with complete medical records were recruited at the center for reproductive medicine and gynecological outpatient department, Xuzhou Central Hospital (outpatient consultation) from July 2012 to December 2020. Any medications known to affect sex hormone, glucose or lipid metabolism were discontinued for at least three months before the study. The study was approved by the Institutional Review Board of Xuzhou Central Hospital. Informed consent was obtained either from a legal guardian of each subject younger than 18 years old or from those subjects who were 18 years old or older.

PCOS adults (aged 20-41 years) were diagnosed when meeting at least two out of the following three criteria [4] (PCOS adolescents (aged 13-19 years) required the presence of all three criteria) [21]: (i) oligand/or anovulation (i.e. eight or fewer menstrual cycles in a year or menstrual cycles more than 35 days in length) (ii) clinical hyperandrogenism (i.e. acne or modified Ferriman-Gallwey scores $\geq 8$ ) or 
biochemical hyperandrogenism (i.e. total testosterone $(T T) \geq 2.6 \mathrm{nmol} / \mathrm{L}$, free testosterone $(\mathrm{FT}) \geq 6.0$ $\mathrm{pg} / \mathrm{mL}$ ); and (iii) polycystic ovaries (i.e. presence of $\geq 12$ follicles in each ovary measuring 2-9 $\mathrm{mm}$ in diameter) and exclusion of related disorders (e.g. congenital adrenal hyperplasia, androgen-secreting tumours and Cushing's syndrome, hyperprolactinemia, thyroid disorders).

Glucose tolerance was determined using the following indicators [3]: normal fasting plasma glucose (FPG) $(\mathrm{FPG}<6.1 \mathrm{mmol} / \mathrm{L})$; impaired fasting glucose $(\mathrm{IFG})=\mathrm{FPG} \geq 6.1 \mathrm{mmol} / \mathrm{L}$ but $<7.0 \mathrm{mmol} / \mathrm{L}$; normal glucose tolerance $(N G T)=2$-h post-oral glucose load $(2 \mathrm{~h}-\mathrm{PG})<7.8 \mathrm{mmol} / \mathrm{L} ; \mathrm{IGT}=2 \mathrm{~h}-\mathrm{PG} \geq 7.8 \mathrm{mmol} / \mathrm{L}$ but $<11.1 \mathrm{mmol} / \mathrm{L}$; and T2DM = FPG $\geq 7.0 \mathrm{mmol} / \mathrm{L}$ or $2 \mathrm{~h}-\mathrm{PG} \geq 11.1 \mathrm{mmol} / \mathrm{L}$; prediabetes = IFG or IGT; $A G T=2 \mathrm{~h}-\mathrm{PG} \geq 7.8 \mathrm{mmol} / \mathrm{L}$.

\section{Study Protocol}

All the recruited patients underwent 75-g OGTT and anthropometric measurements. All parameters were measured, as previously described [22].

Fasting blood samples were obtained from PCOS patients between the first and fifth day of menstrual period/withdrawal bleeding. Prolactin, TT, FT, and thyroid-stimulating hormone were assessed by chemiluminescence immunometric assay (Beckman Unicel Dxl 800; Abbott Immulite 2000 analyzer). 17ahydroxyprogesterone was measured using the ELISA method. Plasma glucose was measured using glucose oxidase method (Hitachi 7600 autoanalyzer). Plasma insulin was measured using chemiluminescence immunometric assay (Roche e601 analyzer). Total cholesterol (CHOL), triglycerides (TG), HDL-C, and low-density lipoprotein cholesterol (LDL-c) were measured using enzymatic colorimetric method (Hitachi 7600 autoanalyzer).

Indices were evaluated based on the formulas presented below:

$\mathrm{BMI}=$ weight $(\mathrm{kg}) /$ height $(\mathrm{m})^{2}$

$\mathrm{LAP}=[\mathrm{WC}(\mathrm{cm})-58] \times \mathrm{TG}(\mathrm{mmol} / \mathrm{L}) .[23]$

CVAl $=-187.32+1.71 \times$ age $+4.23 \times \mathrm{BMI}+1.12 \times \mathrm{WC}(\mathrm{cm})+39.76 \times \log 10[\mathrm{TG}(\mathrm{mmol} / \mathrm{L})]-11.66 \times$ $\mathrm{HDL}(\mathrm{mmol} / \mathrm{L})[16]$

$\mathrm{VAl}=[\mathrm{WC}(\mathrm{cm}) /[36.58+(1.89 \times \mathrm{BMI})]] \times[\mathrm{TG}(\mathrm{mmol} / \mathrm{L}) / 0.81] \times[1.52 / \mathrm{HDL}-\mathrm{C}(\mathrm{mmol} / \mathrm{L})][16]$

TyG $=\operatorname{Ln}[$ fasting triglycerides $(\mathrm{mg} / \mathrm{dL}) \times$ fasting glucose $(\mathrm{mg} / \mathrm{dL}) / 2][9]$

\section{Statistical Analysis}

The data were analyzed by the statistical software SPSS version 24.0 for Windows. We assessed the normality of the distribution of all continuous variables using the Kolmogorov-Smirnov test. As the variables were not normally distributed, continuous variables were described as median with 25 th -75 th 
percentile, and the differences between the groups were determined by the Mann-Whitney $\mathrm{U}$ test.

Receiver operating characteristic (ROC) curves and the area under ROC curves (AUCs) were used to assess the accuracy of each index in detecting AGT. Differences in AUCs were assessed by the method described by Hanley and McNeil. $P \leq 0.05$ (two-tailed) was considered statistically significant.

\section{Results}

In the overall study population, the prevalence of alterations in glucose metabolism was as follows: IFG 39/1113 (3.50\%), IGT 202/1113 (18.15\%), and T2DM 47/1113 (4.30\%).

Of the 47 women with T2DM, 5 were diagnosed based solely on FPG level, 34 on $2 \mathrm{~h}-\mathrm{PG}$, and 8 based on composite FPG and 2 h-PG. AGT was observed in 244 women $(244 / 1113,21.92 \%)$, the remaining 869 women $(869 / 1113,78.08 \%)$ demonstrated NGT.

As shown in Table 1, there were higher levels of age, BMI, WC, FPG, FIN, TG, LDL-C, CHOL, FT, LAP, VAl, CVAI, and TyG in subjects with AGT compared to those with NGT, while HDL-c was found lower in the same subjects $(P<0.05)$. No significant difference in TT $(P>0.05)$ was noticed. 
Table 1

Clinical and laboratory characteristics of PCOS patients with AGT and NGT (median [range])

\begin{tabular}{|lllc|}
\hline & NGT(n=869) & AGT(n=244) & $P$ \\
\hline Age (years) & $26.00(22.00-29.00)$ & $29.00(26.00-32.00)$ & $<0.001$ \\
\hline BMI (kg/m²) & $21.23(19.22-24.14)$ & $25.41(22.57-28.60)$ & $<0.001$ \\
\hline WC (cm) & $73.00(68.00-81.00)$ & $84.00(76.00-92.00)$ & $<0.001$ \\
\hline FPG (mmol/L) & $5.00(4.70-5.30)$ & $5.30(5.00-5.80)$ & $<0.001$ \\
\hline FIN(mU/L) & $7.10(3.90-11.30)$ & $11.30(7.45-17.40)$ & $<0.001$ \\
\hline TG (mmol/L) & $1.03(0.74-1.38)$ & $1.60(1.05-2.21)$ & $<0.001$ \\
\hline HDL-c (mmol/L) & $1.54(1.33-1.80)$ & $1.34(1.16-1.58)$ & $<0.001$ \\
\hline LDL-c (mmol/L) & $2.83(2.34-3.35)$ & $3.13(2.59-3.65)$ & $<0.001$ \\
\hline CHOL (mmol/L) & $4.75(4.30-5.44)$ & $5.21(4.51-5.73)$ & $<0.001$ \\
\hline LAP & $14.96(7.70-30.40)$ & $41.36(21.80-65.14)$ & $<0.001$ \\
\hline VAI & $1.18(0.78-1.85)$ & $2.17(1.26-3.48)$ & $<0.001$ \\
\hline CVAl & $10.90(-11.46-40.36)$ & $57.85(33.90-84.25)$ & $<0.001$ \\
\hline TyG & $8.31(7.99-8.64)$ & $8.84(8.39-9.23)$ & $<0.001$ \\
\hline TT (nmol/L) & $2.26(1.65-2.90)$ & $2.18(1.62-2.88)$ & 0.189 \\
\hline FT (pg/mL) & $3.02(1.96-4.70)$ & $3.64(2.438-5.83)$ & 0.001 \\
\hline $\begin{array}{l}\text { BMl, body mass index; WC, waist circumference; FPG, fasting plasma glucose; FIN, fasting insulin; TG, } \\
\text { triglycerides; HDL-c, high-density lipoprotein cholesterol; LDL-C, low-density lipoprotein cholesterol; }\end{array}$ \\
\hline $\begin{array}{l}\text { CHOL, total cholesterol; LAP, lipid accumulation product; VA, visceral adiposity index; CVAl, Chinese } \\
\text { visceral adiposity index; TyG, triglyceride glucose Index; FT, free testosterone; TT, total testosterone }\end{array}$ \\
\hline
\end{tabular}

Table 2 presents the correlation coefficients between the $2 \mathrm{~h}-\mathrm{PG}$ and each index. A significant positive correlation between the $2 \mathrm{~h}-\mathrm{PG}$ and FPG, BMI, WC, LAP, VAl, CVAl, TyG $(P<0.05)$ was observed. The $2 \mathrm{~h}-\mathrm{PG}$ and CVAI $(r=0.469)$ revealed the strongest correlation. 
Table 2

Spearman rank correlations between the $2 \mathrm{~h}-\mathrm{PG}$ and FPG, obesity-and lipid-related indices.

\begin{tabular}{|lll|}
\hline & $\mathbf{r}$ & $\boldsymbol{p}$ \\
\hline FPG & 0.381 & $<0.001$ \\
CVAl & 0.469 & $<0.001$ \\
VAI & 0.400 & $<0.001$ \\
LAP & 0.436 & $<0.001$ \\
TyG & 0.426 & $<0.001$ \\
BMI & 0.412 & $<0.001$ \\
WC & 0.383 & $<0.001$ \\
\hline 2 h-PG, 2-h post-oral glucose load; FPG, fasting plasma glucose; CVAl, Chinese visceral adiposity \\
index; VAl, visceral adiposity index; LAP, lipid accumulation product; TyG, triglyceride glucose Index; \\
BMI, body mass index; WC, waist circumference
\end{tabular}

In this study, ROC curves analysis indicated that all indices could predict AGT and CVAl, showing the highest AUC, followed by LAP, BMI, TyG, VAI, WC, and FPG. The differences in AUC between CVAI with other indices were statistically significant $(P<0.05)$ (Table 3, Figure1).

Table 3

ROC curves data for detecting AGT using each indicator

\begin{tabular}{|lllllll|}
\hline & AUC $(\mathbf{9 5 \%} \mathrm{Cl})$ & Cut-off point & Sensitivity & Specificity & Youden index & $P$ \\
\hline BMI & $0.725(0.689-0.762)$ & 22.70 & 0.721 & 0.677 & 0.398 & $<0.001$ \\
WC & $0.709(0.671-0.746)$ & 80.5 & 0.635 & 0.734 & 0.369 & $<0.001$ \\
FPG & $0.695(0.655-0.734)$ & 5.35 & 0.492 & 0.796 & 0.288 & 0.006 \\
LAP & $0.736(0.700-0.772)$ & 26.19 & 0.705 & 0.689 & 0.394 & 0.004 \\
CVAl & $0.758(0.722-0.793)$ & 32.61 & 0.734 & 0.699 & 0.432 & reference \\
VAI & $0.708(0.671-0.746)$ & 1.85 & 0.582 & 0.748 & 0.330 & 0.001 \\
TyG & $0.724(0.687-0.760)$ & 8.71 & 0.566 & 0.793 & 0.358 & 0.037 \\
\hline
\end{tabular}

$P$. CVAl vs other indices

BMI, body mass index; WC, waist circumference; FPG, fasting plasma glucose; CVAl, Chinese visceral adiposity index; VAI, visceral adiposity index; LAP, lipid accumulation product; TyG, triglyceride glucose Index 


\section{Discussion}

In our study, we directly compared the performance of FPG, CVAI, VAI, LAP, TyG, WC, and BMI in detecting AGT and observed FPG demonstrated the lowest AUC value (0.695) and the lowest sensitivity (49.2\%) among all the indices for detecting AGT. Thus, although FPG is an inexpensive assay and does not require mathematical calculations, it may not reliably detect AGT in our PCOS population, a finding consistent with previous studies [3,5].

We next evaluate the abilities of six obesity- and lipid-related indices (CVAI, VAl, LAP, TyG, BMI, WC) as indicators of detecting AGT in PCOS patients. In line with $2 \mathrm{~h}-\mathrm{PG}$ as criteria for AGT, our data demonstrated that CVAl outperformed other indices with a higher correlation coefficient and a larger AUC for AGT detection in Chinese women with PCOS.

CVAl, is known as Chinese VAl. VAl, was introduced to estimate visceral adipose function for the Caucasians and is an index established with the use of BMI, WC, TG, and HDL-C [10,24]. In Caucasians, VAl was used as a valuable indicator of visceral adiposity and adipose tissue dysfunction to predict the risk for cardiovascular diseases and insulin resistance [24]. However, a previous study showed that VAI was poorly associated with adipose tissue area and has poor diagnostic performance to predict prediabetes and diabetes in Chinese [16]. In conformity with previous findings, we showed that the AUC of VAl for AGT was 0.708, which was lower than the AUC of CVAI (0.758), LAP (0.736), TyG (0.724), and BMI (0.725). Moreover, data based on correlation analyses also revealed similar tendencies. The main reason of the discrepancy may be ethnic difference and subject characteristic. VAl was developed initially for Caucasians. However, compared to equivalent Caucasians, Asian subjects have a greater proportion of body fat for a given BMI level [25] and are more prone to accumulate fat around the abdomen [26].

The CVAl index based on the combination of age and all the parameters of VAI (BMI, WC, TG and HDL-C) was developed to estimate visceral fat area for Chinese by Xia and colleagues [16]. In their study, among 6495 middle-aged and elderly Chinese, CVAl provided higher AUCs for the diagnosis of T2DM and prediabetes than BMI, WC and VAI [16]. Also, two prospective studies indicated that CVAI proved a better predictor of prediabetes and diabetes compared to BMI, WC, and VAI in the general Chinese population $[15,27]$. However, no data are currently available establishing any association between CVAI and AGT. In our cohort of PCOS patients, CVAl provided the strongest correlation with $2 \mathrm{~h}-\mathrm{PG}$ and the largest AUC for detecting AGT, thereby suggesting that CVAl might be a more useful indicator of AGT than LAP, TyG, BMI, VAI, and WC in Chinese women with PCOS. Although the calculation of CVAI requires more parameters and more complicated formulas, the parameters (age, BMI, WC, TG, and HDL-C) are easily available and low cost in clinical practice, and the complex algorithms can easily be performed by Tablets or Smartphone. In our study population, ROC curves analysis suggested a threshold value of 32.61 in CVAI for the detection of AGT (73.4\% sensitivity, $69.9 \%$ specificity, and 0.758 AUC).

LAP, a novel index based on a combination of WC and TG, was first introduced for the U.S. National Health and Nutrition Examination Survey [23]. Furthermore, it is proposed as a valuable marker indicating T2DM and prediabetes $[9,12]$. However, few studies have explored the association of LAP with AGT. The 
results of previous studies indicated that LAP performed better than BMI in identifying abnormal glucose regulation in the young Korean women and provided higher AUC in estimating the risk for IGT than BMI and waist-to-hip ratio in Austrian PCOS women [12,19], which was accordance with our results. However, these studies did not compare the utility of LAP with other novel obesity- and lipid-related indices. Our analysis showed that LAP performed superior to TyG, VAI, BMI, and WC, but inferior to CVAl in detecting AGT in Chinese PCOS population. According to the maximized Youden index, the LAP 26.19 (with AUC 0.736 , sensitivity $70.5 \%$, specificity $68.9 \%$ ) was found to be the cut-off point for AGT in our study population, which was much higher than that in Korean PCOS women (12.98) [20]. This discrepancy could be partially due to ethnic difference.

TyG index, a composite indicator composed of TG and FPG, is a key predictor of T2DM and prediabetes in severl epidemiological studies. The TyG performed better than homeostasis model assessment of insulin resistance (HOMA-IR) and TG/HDL-c in predicting T2DM in Korean adults without initial T2DM [28]. A study conducted among 3307 elderly Colombian individuals suggested better discriminative power of the TyG index than BMI, WC, and VAI to predict prediabetes [11]. However, OGTT was not performed, and prediabetes was defined as a FPG of 100 to $125 \mathrm{mg} / \mathrm{dL}$ ( 5.6 to $7.0 \mathrm{mmol} / \mathrm{L}$ ) in this study. In a prospective cohort study conducted on 4543 Chinese individuals without initial prediabetes or diabetes, Wen et al. reported that TyG provided a larger AUC for predicting prediabetes and isolated IGT than FPG, WC, and BMI [8]. However, this study did not compare the ability of TyG with other novel obesity- and lipidrelated indices. In the current study, TyG was found inferior to CVAI and LAP for detecting AGT based on AUC and correlation coefficient.

Due to the fact that a plasma insulin assay is not yet available in all laboratories, has poor reproducibility and is costly [29], we do not evaluate the performance of the indices those using insulin for detecting AGT. Indeed, they do not perform better than obesity- and lipid-related indices studied in our report (data not shown).

Despite these relevant findings, it is important to point out the limitation of our study. All the subjects were recruited from Xuzhou Central Hospital, and only those with complete medical records. This population may not represent all the Chinese women with PCOS, with a possibility of bias in the results.

In conclusion, CVAl performed better than LAP, TyG, VAl, BMI, WC, and FPG for detecting AGT in Chinese women with PCOS. However, due to the lower sensitivity and specificity of CVAl, it may be used as a screening indicator of AGT in Chinese women with PCOS when it becomes unfeasible to conduct an OGTT.

\section{Declarations}

\section{Data Availability}

The data used to support the findings of this study are available from the corresponding author upon request. 


\section{Conflict of Interest}

The authors declared no conflict of interest.

\section{Authors' contributions}

Q. Q. Yin performed the study, analyzed and interpreted the data, and drafted the paper. X. N. Yan collected and analyzed the data. Y. J. Cao collected the data and revised the draft. J. H. Zheng designed, performed the study, and revised the paper.

\section{Ethics Statement}

The study was approved by the institutional review board of Xuzhou Central Hospital.

\section{Acknowledgments}

This study was supported by grants from National Natural Science Foundation of China (81571405), Jiangsu Province natural science foundation of China (BK20161169).

\section{Disclosure of interest}

The authors report no conflict of interest.

\section{References}

1. Han M., Qin P., Li Q., et al.: Chinese visceral adiposity index: A reliable indicator of visceral fat function associated with risk of type 2 diabetes. Diabetes Metab Res Rev. 37, e3370 (2021)

2. Wei J., Liu X., Xue H., et al.: Comparisons of Visceral Adiposity Index, Body Shape Index, Body Mass Index and Waist Circumference and Their Associations with Diabetes Mellitus in Adults. Nutrients. 11,1580 (2019)

3. Chen X., Yang D., Li L., et al.: Abnormal glucose tolerance in Chinese women with polycystic ovary syndrome. Hum Reprod. 21, 2027-2032 (2006)

4. Ni RM., Mo Y., Chen X., et al.: Low prevalence of the metabolic syndrome but high occurrence of various metabolic disorders in Chinese women with polycystic ovary syndrome. Eur $\mathrm{J}$ Endocrinol. $161,411-418$ (2009)

5. Ortiz-Flores AE, Luque-Ramírez M, Fernández-Durán E, et al.: Diagnosis of disorders of glucose tolerance in women with polycystic ovary syndrome (PCOS) at a tertiary care center: fasting plasma glucose or oral glucose tolerance test? Metabolism. 93, 86-92 (2019)

6. Legro RS, Kunselman AR, Dodson WC, et al.: Prevalence and predictors of risk for type 2 diabetes mellitus and impaired glucose tolerance in polycystic ovary syndrome: a prospective, controlled study in 254 affected women. J Clin Endocrinol Metab. 84, 165-169 (1999) 
7. Yin Q, Zheng J, Cao Y, et al.: Evaluation of Novel Obesity and Lipid-Related Indices as Indicators for the Diagnosis of Metabolic Syndrome and Premetabolic Syndrome in Chinese Women with Polycystic Ovary Syndrome. Int J Endocrinol. 2021, 7172388 (2021)

8. Wen J, Wang A, Liu G, et al.: Elevated triglyceride-glucose (TyG) index predicts incidence of Prediabetes: a prospective cohort study in China. Lipids Health Dis. 19, 226 (2020)

9. Ahn N, Baumeister SE, Amann U, et al.: Visceral adiposity index (VAl), lipid accumulation product (LAP), and product of triglycerides and glucose (TyG) to discriminate prediabetes and diabetes. Sci Rep. 9, 9693 (2019)

10. Yang J, Wang F, Wang J, et al.: Using different anthropometric indices to assess prediction ability of type 2 diabetes in elderly population: a 5 year prospective study. BMC Geriatr. 18, 218 (2018)

11. Ramírez-Vélez R, Pérez-Sousa MÁ, González-Ruíz K, et al.: Obesity- and Lipid-Related Parameters in the Identification of Older Adults with a High Risk of Prediabetes According to the American Diabetes Association: An Analysis of the 2015 Health, Well-Being, and Aging Study. Nutrients. 11, 2654 (2019)

12. Oh JY, Sung YA, Lee HJ.: The lipid accumulation product as a useful index for identifying abnormal glucose regulation in young Korean women. Diabet Med. 30, 436-442 (2013)

13. Wang Z, He S, Chen X.: Capacity of different anthropometric measures to predict diabetes in a Chinese population in southwest China: a 15-year prospective study. Diabet Med. 36, 1261-1267 (2019)

14. Tohidi M, Baghbani-Oskouei A, Ahanchi NS, et al.: Fasting plasma glucose is a stronger predictor of diabetes than triglyceride-glucose index, triglycerides/high-density lipoprotein cholesterol, and homeostasis model assessment of insulin resistance: Tehran Lipid and Glucose Study. Acta Diabetol. 55, 1067-1074 (2018)

15. Wu J, Gong L, Li Q, et al.: A Novel Visceral Adiposity Index for Prediction of Type 2 Diabetes and Prediabetes in Chinese adults: A 5-year prospective study. Sci Rep. 7, 13784 (2017)

16. Xia MF, Chen Y, Lin HD, et al.: A indicator of visceral adipose dysfunction to evaluate metabolic health in adult Chinese. Sci Rep. 6, 38214 (2016)

17. Oh JY, Sung YA, Lee HJ.: The visceral adiposity index as a predictor of insulin resistance in young women with polycystic ovary syndrome.Obesity (Silver Spring). 21, 1690-1694 (2013)

18. Malavazos AE, Cereda E, Ermetici F, et al.: The "lipid accumulation product" is associated with 2-hour postload glucose outcomes in overweight/obese subjects with nondiabetic fasting glucose. Int $\mathrm{J}$ Endocrinol. 2015, 836941 (2015)

19. Wehr E, Gruber HJ, Giuliani A, et al. The lipid accumulation product is associated with impaired glucose tolerance in PCOS women. J Clin Endocrinol Metab. 96, E986-E990 (2011)

20. Jeong K, Park SJ, Jeon JH, et al.: Predictive markers for abnormal glucose intolerance in women with polycystic ovary syndrome. Minerva Med. 107, 185-193 (2016)

21. Ozler S, Oztas E, Tokmak A, et al.: The association of thiol/disulphide homeostasis and lipid accumulation index with cardiovascular risk factors in overweight adolescents with polycystic ovary syndrome. Clin Endocrinol (Oxf). 84, 516-523 (2016) 
22. Hatch R, Rosenfield RL, Kim MH, et al. Hirsutism: implications, etiology, and management. Am J Obstet Gynecol. 140(, 815-830 (1981)

23. Kahn HS.: The "lipid accumulation product" performs better than the body mass index for recognizing cardiovascular risk: a population-based comparison. BMC Cardiovasc Disord. 5, 26 (2005)

24. Amato MC, Giordano C, Galia M, et al.: Visceral Adiposity Index: a reliable indicator of visceral fat function associated with cardiometabolic risk. Diabetes Care. 33, 920-922 (2010)

25. Deurenberg P, Deurenberg-Yap M, Guricci S. Asians are different from Caucasians and from each other in their body mass index/body fat percent relationship. Obes Rev. 3, 141-146 (2002)

26. Nazare JA, Smith JD, Borel AL, et al.: Ethnic influences on the relations between abdominal subcutaneous and visceral adiposity, liver fat, and cardiometabolic risk profile: the International Study of Prediction of Intra-Abdominal Adiposity and Its Relationship With Cardiometabolic Risk/Intra-Abdominal Adiposity. Am J Clin Nutr. 96, 714-726 (2012)

27. Xia MF, Lin HD, Chen LY, et al.: Association of visceral adiposity and its longitudinal increase with the risk of diabetes in Chinese adults: A prospective cohort study. Diabetes Metab Res Rev. 34, e3048 (2018)

28. Lee SH, Kwon HS, Park YM, et al.: Predicting the development of diabetes using the product of triglycerides and glucose: the Chungju Metabolic Disease Cohort (CMC) study. PLoS One. 9, e90430 (2014)

29. Kang B, Yang Y, Lee EY, et al.: Triglycerides/glucose index is a useful surrogate marker of insulin resistance among adolescents. Int J Obes (Lond). 41, 789-792 (2017)

\section{Figures}




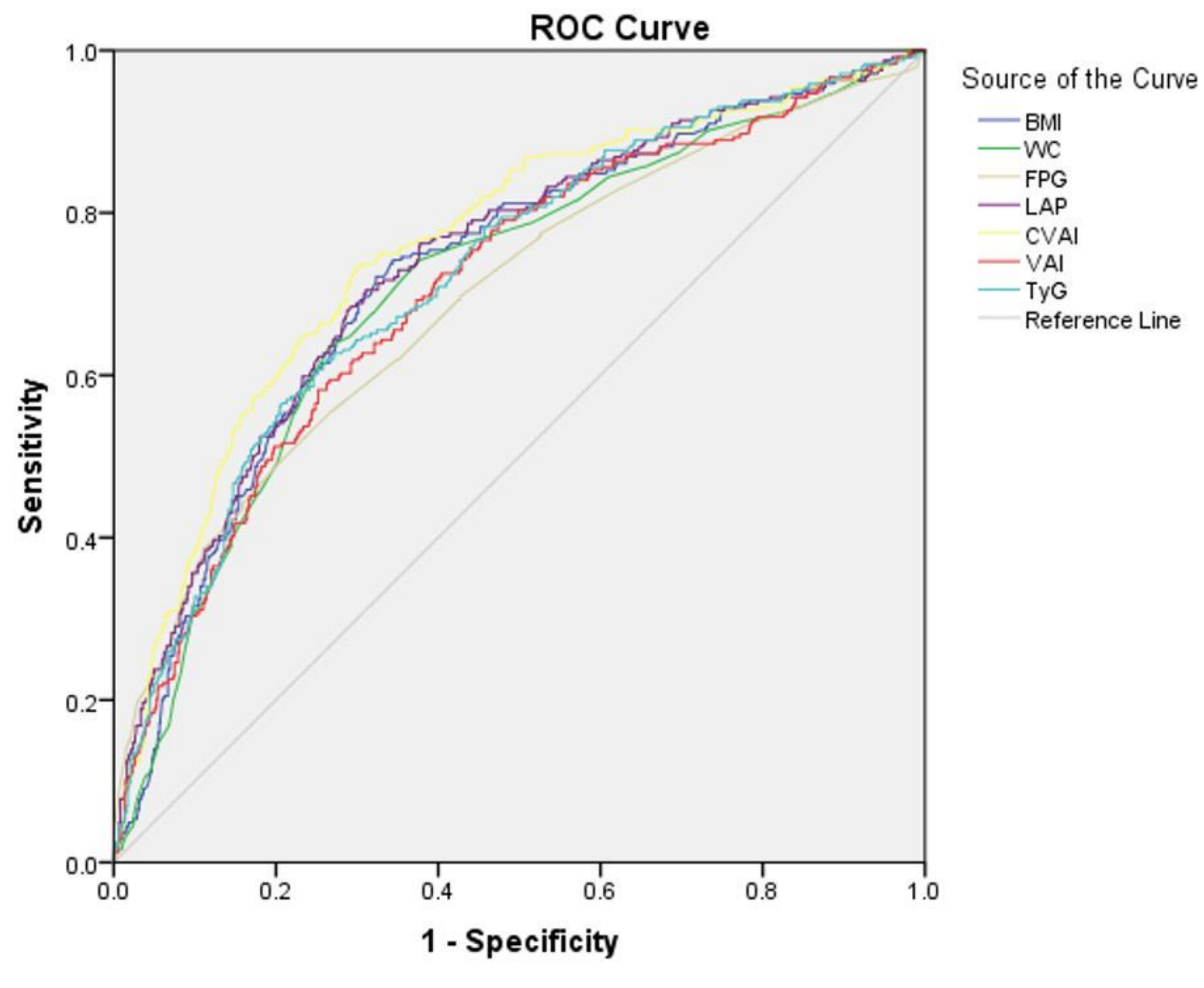

Diagonal segments are produced by ties.

\section{Figure 1}

Receiver operating characteristic (ROC) curves for each indicator for detecting abnormal glucose tolerance (AGT) in Chinese women with Polycystic ovary syndrome (PCOS). 